\title{
Brady- and Tachycardia in Light of the Valsalva and the Mueller Maneuver (Apnea)
}

\author{
Poul-Erik Paulev, ${ }^{*}$ Yoshiyuki Honda, ${ }^{* *}$ Yoshikazu SaKaKibara, ${ }^{* *}$ \\ Tsuguo Morikawa, ${ }^{* *}$ Yukio Tanaka, ${ }^{* *}$ \\ and Wataru NaKAMURA** \\ * Institute of Medical Physiology B, University of Copenhagen, \\ Sports/Cybernetics Panum, \\ Blegdamsvey 3, 2200 Copenhagen N, Denmark \\ ** Department of Physiology, School of Medicine, \\ Chiba University, Chiba, 280 Japan
}

\begin{abstract}
With a computerized impedance cardiograph we measured stroke volume (sv), cardiac output and heart rate (HR) in four men, during apnea with positive or negative intrapulmonic pressure (i.e., Valsalva and Mueller maneuver) in air. During Valsalva maneuvers the sv was reduced, and the compensatory rise in HR failed to keep the cardiac output at the control level before apnea. During both types of apnea, the diastolic pressure was increased as was the total peripheral resistance (TPR). The vasoconstriction and tachycardia during Valsalva maneuvers can be explained as a sino-aortic baroreceptor phenomenon in man. The smaller changes occurring during Mueller maneuvers result in no change in the transmural arterial pressure in the thorax, compared to the control level. Thus, without a stimulus there is no change in heart rate. The alveolar oxygen uptake and carbon dioxide elimination during apnea at total lung capacity was much larger than in the control phase before both types of apnea. The arteriolar vasoconstriction with increased TPR during the Valsalva apnea, was accompanied by a reduction in the stroke work of the left ventricle to approximately $50 \%$ of the work in the control phase.
\end{abstract}

Key words: apnea, breath-holding, bradycardia, tachycardia, baroreceptors.

Alteration of the cardiac rhythm during diving and during apnea in air (i.e., breath-holding) have been found in several animals (IRVING, 1964). Besides these alterations, changes in vascular resistance of organs and limbs also occur-changes which reserve the reduced cardiac output and the limited oxygen stores for those

Received for publication March 1, 1988

* To whom all correspondence should be addressed. 
organs (i.e., brain and heart) most dependent on a continuous supply of oxygenated blood (ElSNER and ScHOlANDER, 1965). These phenomena are known as an "oxygen conserving reflex," but the physiological mechanisms involved are not fully explained. Nevertheless, it is generally assumed that the mechanism by which "diving bradycardia" of man and the related oxygen conservation (i.e., organspecific prolonged utilization of the oxygen stores) is brought about, is similar to the "oxygen conserving reflex" in aquatic mammals (OLSEN et al., 1962; BLIX and FolKow, 1983; HoNG, 1987). In man, the cardiovascular reactions to apnea depend upon the intrapulmonic pressure (i.e. the airway pressure) or the pressure in the alveolar air (PAULEv, 1968). The physiological mechanism is related to the high pressure baroreceptor control system (PAULEV, 1969). Although there is no direct evidence of any oxygen conservation, neither during apnea, nor diving or face immersion, such a "conservation" may still be important in emergency situations. The object of the present study was to measure stroke volume (sv), cardiac output $(\dot{Q})$, mean arterial blood pressure (MAP), total peripheral resistance (TPR), and pulmonary net gas transfer during apnea, with a positive intrapulmonic pressure (i.e., Valsalva maneuver), and with a negative pressure (i.e., Mueller maneuver). The aim was to explain the cardiovascular alterations at the two apnea maneuvers and measure a possible oxygen conservation in man.

\section{METHODS}

Subjects. Four normal males were studied (Table 1). The subjects were found among the authors; they had no history or current symptoms of cardiopulmonary disease. Informed consent was obtained. The protocol was approved by the local ethics committee.

Recording and equipment. All recordings were conducted in a quiet room with the subjects seated. Four stainless steel strips of $5 \mathrm{~mm}$ width and of $0.1 \mathrm{~mm}$ thickness were arranged around the neck and the chest wall of the subject, constituting the tetrapolar electrode system of an impedance cardiograph. Stroke volume, heart rate, and cardiac output were automatically determined from the electrode signals

Table 1. Characteristics of the subjects.

\begin{tabular}{ccccc}
\hline Subject & $\begin{array}{c}\text { Age } \\
\text { (Year) }\end{array}$ & $\begin{array}{c}\text { Height/weight } \\
(\mathrm{m} / \mathrm{kg})\end{array}$ & $\begin{array}{c}\text { Vital capacity } \\
\text { (VC, } / \text { STPD) }\end{array}$ & $\begin{array}{c}\text { Total lung capacity } \\
\text { (TLC, } \text { l STPD) }\end{array}$ \\
\hline TM & 33 & $1.73 / 65$ & 4.56 & $6.84^{*}$ \\
WN & 35 & $1.75 / 71$ & 4.89 & $6.35^{*}$ \\
YH & 60 & $1.63 / 71$ & 3.80 & $5.85^{*}$ \\
PP & 51 & $1.78 / 71$ & 4.90 & $6.37^{* *}$ \\
\hline
\end{tabular}

${ }^{*}$ Residual volume (RV) was measured by the helium dilution method. ${ }^{* *} \mathrm{RV}$ was assumed to be $30 \%$ of VC. 
with a Minnesota Impedance cardiograph (model 304-A), and the ECG was continuously monitored with a San-ei cardiosuper (2E 31A). The circulatory variables were continuously processed by a microcomputer on-line system. Triggered by the ECG R-wave, the computer sampled the first time derivative $(\mathrm{d} Z / \mathrm{d} t)$ and basic transthoracic impedance $\left(Z_{\mathrm{o}}\right)$, at a rate of 200 times per s. The sampling was repeated for 5 to 12 cardiac cycles to obtain an average waveform with a favorable signal-to-noise ratio for computer processing. The system is described elsewhere (Мгуното et al., 1981, 1982) and its validity confirmed by comparison with the indirect Fick principle, and also with the thermodilution principle (MuZi et al., 1985). The whole body hematocrit was assumed to be $43 \%$ in these healthy subjects.

Respiratory flow was measured in three subjects with a respiratory hot-wire flowmeter (FR-2, Minato Medical Sci. Co., Ltd.) and end-expiratory gas concentrations were measured with an expired gas monitor (San-ei, 1H 21A) using the infrared (carbon dioxide) and the polarographic (oxygen) principle. The intrapulmonic pressure was measured at the mouth with a mercury manometer, and the arterial blood pressure with a cuff covering the brachial artery (Copal digital sphygmomanometer UA-251). All direct or computed data were recorded on a multichannel recorder (San-ei, RECTI HORIZ-8K).

Protocol and data treatment. The protocol consisted of $15 \mathrm{~s}$ apnea (i.e., breath-holding) separated by 45 -s spontaneous breathing. Just prior to apnea the subject took a deep inspiration. The lung volume during apnea was considered to be total lung capacity (TLC), but was shown to be at a level around $90 \%$ of vital capacity (VC) as also previously found (PAUlev, 1969). The subject was asked to maintain a positive intrapulmonic pressure during 15-s apnea (Valsalva), then breathe spontaneously for $45 \mathrm{~s}$ and ultimately maintain a negative pressure during 15-s apnea (Mueller).

Part I. The purpose was to measure alterations of heart rate, stroke volume, and cardiac output, during a series of Valsalva and Mueller maneuvers. The stroke volume in the control period $\left(\mathrm{sv}_{\mathrm{C}}\right)$ just prior to apnea and the largest alteration during apnea (either minimum or maximum, $\mathrm{sv}_{\mathrm{A}}$ ), were measured in $\mathrm{ml}$ of blood from the calibrated curves, together with the related control heart rate $\left(\mathrm{HR}_{\mathrm{C}}\right)$, apnea heart rate $\left(\mathrm{HR}_{\mathrm{A}}\right)$, and control cardiac output $\left(\dot{Q}_{\mathrm{C}}\right)$ and apnea cardiac output $\left(\dot{Q}_{\mathrm{A}}\right)$. Either five or more beats were averaged, and the averaging period was recorded in seconds. Paired comparison of consecutive Valsalva and Mueller maneuvers was performed. From the beat-by-beat recording, the lowest and the latest HR was measured. Results from three subjects, who performed all 16 apnea periods, were analyzed.

Part II. The purpose was to measure oxygen uptake, carbon dioxide elimination, and alveolar exchange ratio $(R)$ at rest before apnea and during a typical 15 -s apnea (at intrapulmonic pressures of +5.3 , zero, and $-5.3 \mathrm{kPa}$ ). The alveolar oxygen uptake and the carbon dioxide elimination were calculated according to the alveolar gas exchange equation in the period just before apnea. The net transfer of 
gases from the lungs to the body, during $15 \mathrm{~s}$ of apnea was calculated as the difference between the volume of oxygen $\left(\Delta V_{\mathrm{O}_{2}}\right)$ or carbon dioxide $\left(\Delta V_{\mathrm{CO}_{2}}\right)$ in the lungs after the final inspiration (from the functional residual capacity, FRC), before apnea (VI), and just before the expiration after the same apnea period (VE). Thus we can calculate: $\Delta V_{\mathrm{CO}_{2}}$ or $\Delta V_{\mathrm{O}_{2}}=\left(\mathrm{FRC} \times \mathrm{F}_{\mathrm{A}}+\mathrm{VI} \times \mathrm{F}_{\mathrm{I}}\right)-(\mathrm{FRC}+\mathrm{VE}) \times \mathrm{F}_{\mathrm{A}}{ }^{\prime}$. Here the two alveolar fractions are from the final expiration before $\left(\mathrm{F}_{\mathrm{A}}\right)$ apnea, and from the first expiration after $\left(\mathrm{F}_{\mathrm{A}}{ }^{\prime}\right)$ apnea (PAULEV, 1969).

Part III. The purpose was to measure brachial artery pressure and heart rate, produced by a series of Valsalva maneuvers, alternating with Mueller maneuvers. From the systolic and diastolic arterial pressures, the MAP was calculated as the sum of $1 / 3$ of the pulse pressure and the diastolic pressure. According to the Poiseuille equation, the flow of blood is proportional to perfusion pressure; divided by resistance. The MAP minus a central venous pressure (CVP) of $+2.7 \mathrm{kPa}$, was assumed to be representative of the perfusion pressure during Valsalva, and MAP minus a CVP of zero during Mueller maneuvers as in previous reports (PAulev and WetterQvist, 1968; PAulev, 1969). The TPR was calculated as the perfusion pressure, divided by the cardiac output, and expressed in pressure resistance units (PRU). The stroke work of the left ventricle (W left) was estimated as the perfusion pressure multiplied with stroke volume.

Statistics. The level of significance was chosen as two-sided $\alpha=0.05$. The distribution of the variables was, with good approximation, shown to be normal. Accordingly, statistical comparisons were performed using a paired $t$-test.

\section{RESULTS}

\section{Part I}

A typical recording of sv, HR, and $\dot{Q}$-all averages of five heart beats-is shown together with beat-by-beat recordings of heart rate, ECG, and the first time derivative of the transthoracic impedance, $\mathrm{d} Z / \mathrm{d} t$ (Fig. 1). During the Valsalva maneuver the sv and $\dot{Q}$ typically fell below the control level, whereas during the Mueller maneuver they varied around the level (Fig. 1). A rise in HR is particularly evident in the beat-by-beat curve during apnea, and at the large inspiration just following the Valsalva maneuver. The recording shows an acceptable $\mathrm{d} Z / \mathrm{d} t$ with or without apnea, and the difference between beat-by-beat, and an average of five heart beats (Fig. 1). The ratio between a given variable measured in the apnea period and the last measure in the control period before apnea was calculated for three variables (sv, HR, $\dot{Q}$ ). The ratio between simultaneously recorded stroke volume and heart rate in the apnea period was also calculated. This sv-apnea/HRapnea ratio was used as a less rate dependent expression of the ejection volume during comparable apnea periods (Fig. 2). For each consecutive pair of Valsalva and Mueller maneuvers, these four ratios were plotted (Fig. 2). The Valsalva apnea period is characterized by a definite reduction of sv and $\dot{Q}$ (Fig. $2 \mathrm{~A}, \mathrm{C}$ ), and a rise in HR (Fig. 2B), whereas the Mueller apnea period is characterized by variations 


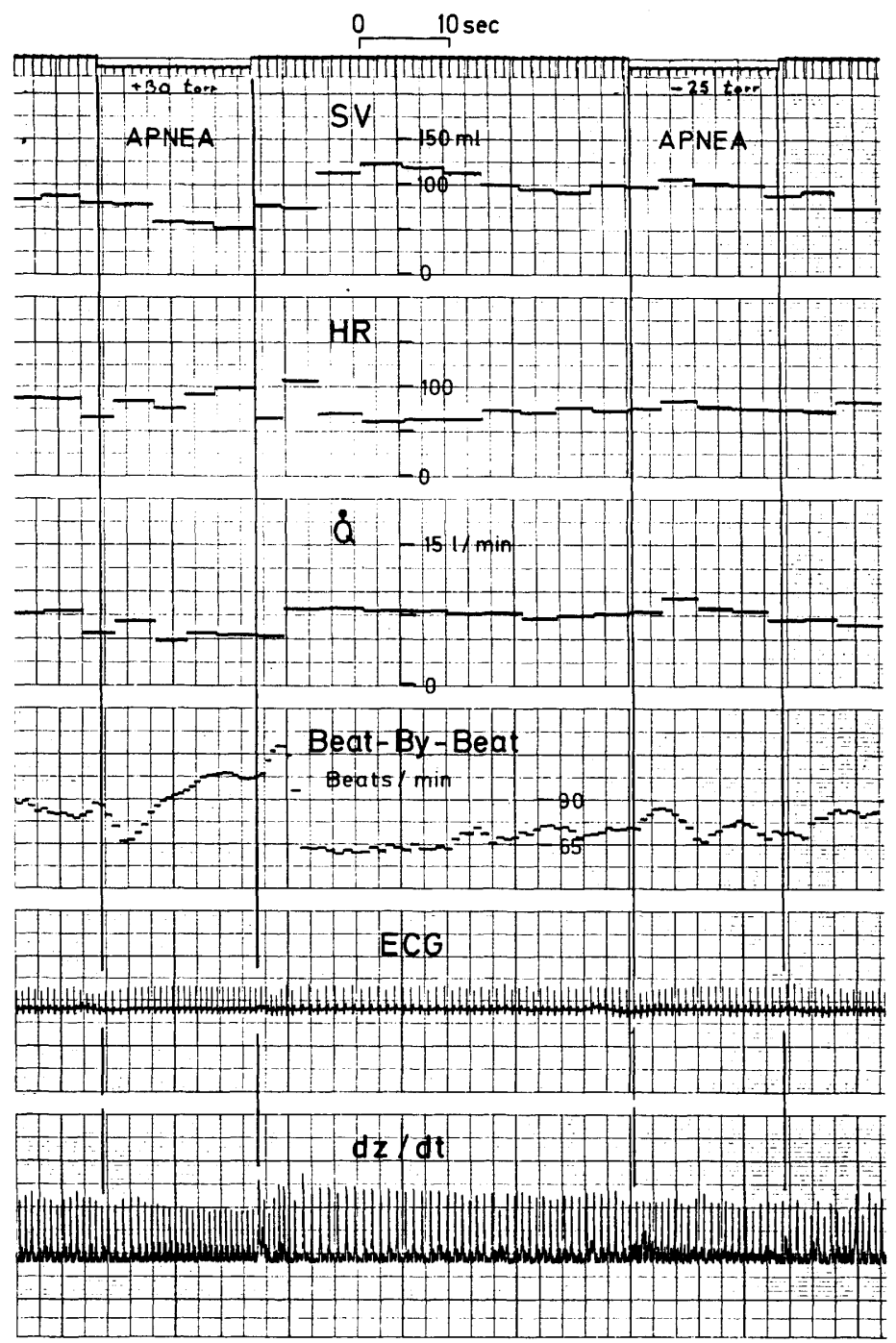

Fig. 1. Original recording from subject TM performing Valsalva and Mueller apnea in air. The upper record is a time axis in seconds with even markings.

around a ratio of 1 (Fig. $2 \mathrm{~A}, \mathrm{~B}, \mathrm{C}$ ). All of the Valsalva events showed reduced Sv (to $50-80 \%$ of the preceding control values), whereas the related Mueller events were characterized by large variations of sv (between 55 and $150 \%$, Fig. 2A). As a measure of the stroke work relative to the average heart beat during apnea, the sv/HR ratio was below one during Valsalva, and between 0.6 and 1.6 during Mueller maneuver (Fig. 2D). This arbitrary estimate of a standardized stroke volume is proportional to the calculated stroke work of the left ventricle (W 
A $0^{(0.71 ; 1.66)}$
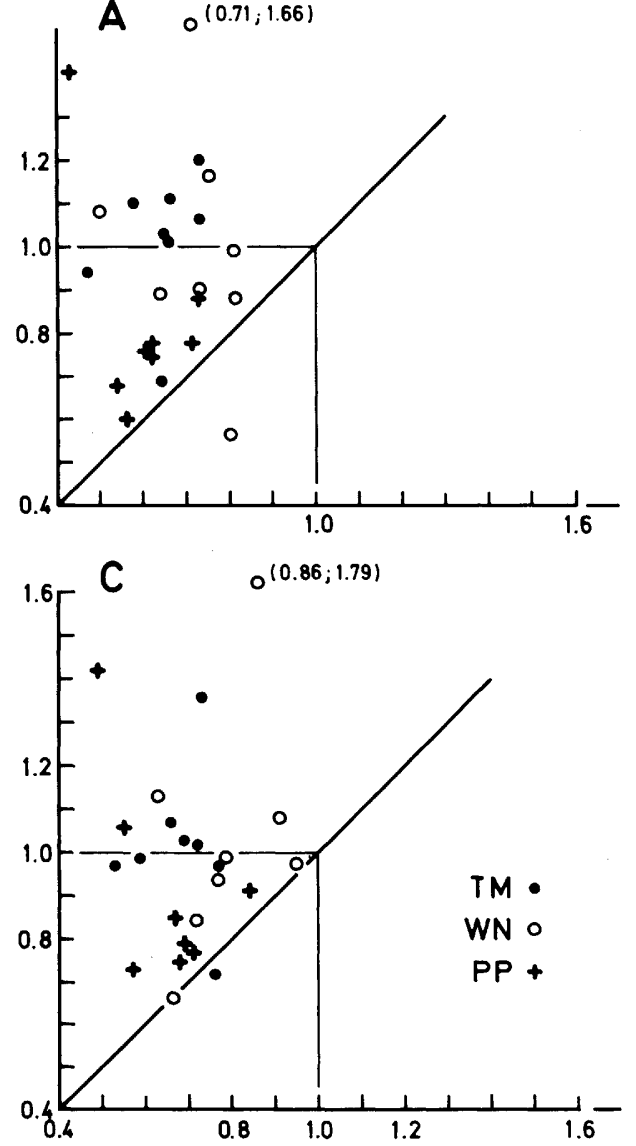

B
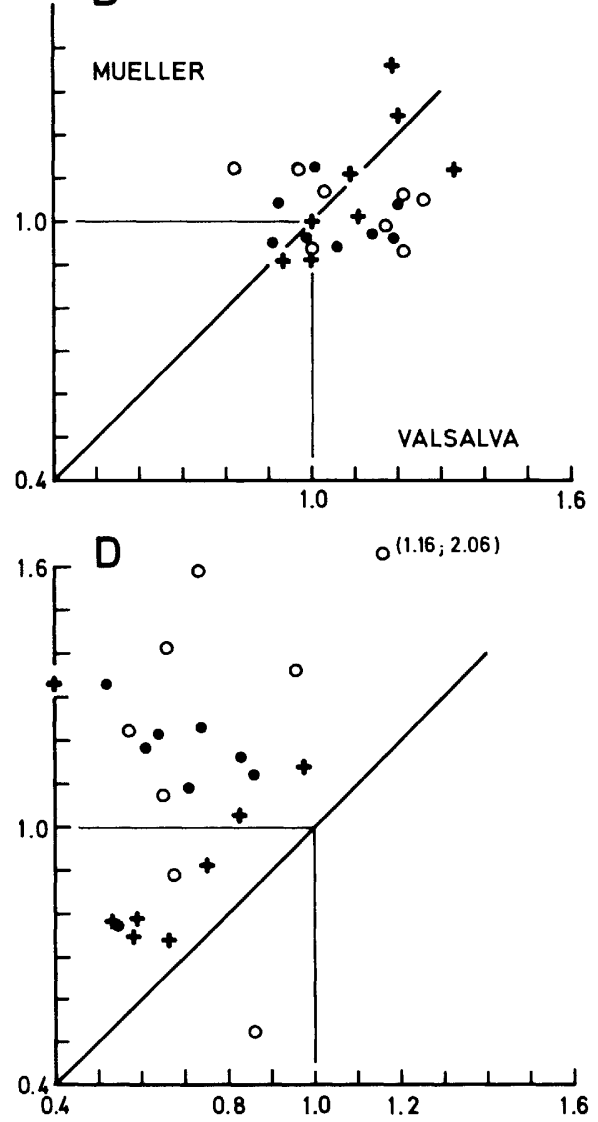

Fig. 2. The four plots are shown with Mueller apnea as the ordinate and with Valsalva apnea as the abscissa. A: the individual stroke volume ratios, B: the heart rate ratios, $\mathrm{C}$ : the cardiac output ratios, and $\mathrm{D}$ : the ratios between stroke volume and heart rate in the apnea period only (sv-apnea/HR-apnea-ratio).

left, Table 3).

\section{Part II}

The oxygen uptake, carbon dioxide elimination, and alveolar exchange ratio $(R)$ in $15 \mathrm{~s}$ of the control period before apnea are compared to the net transport of oxygen and carbon dioxide during 15-s apnea at intrapulmonic pressures of +5.3 , zero, and $-5.3 \mathrm{kPa}$ (Table 2). The net transport of alveolar gases during $15-\mathrm{s}$ apnea is clearly much larger than those in the control phase ( $15 \mathrm{~s}$ before apnea). During all these apnea events (all at 'TLC'), the lung diffusion capacity seems to be increased to such an extent that large quantities of oxygen are transferred from the lungs to the body, and less carbon dioxide is given off from the body to the lungs (negative 
Table 2. Oxygen uptake $\left(\dot{V}_{\mathrm{O}_{2}}\right)$ and carbon dioxide elimination $\left(\dot{V}_{\mathrm{CO}_{2}}\right)$ in the control period before apnea, compared to the size of the gas exchange $\left(V_{\mathrm{O}_{2}}^{\prime}\right.$ and $\left.V_{\mathrm{CO}_{2}}^{\prime}\right)$ during apnea.

\begin{tabular}{|c|c|c|c|c|c|c|c|}
\hline \multirow{2}{*}{ Subject } & \multirow{2}{*}{$\begin{array}{l}\text { Pressure** } \\
\quad(\mathrm{kPa})\end{array}$} & \multicolumn{2}{|c|}{ Oxygen uptake } & \multicolumn{2}{|c|}{ Carbon dioxide } & \multicolumn{2}{|c|}{ Exchange ratio $(R)^{\prime}$} \\
\hline & & $\begin{array}{c}\text { Control } \\
\dot{V}_{\mathrm{O}_{2}}\end{array}$ & $\begin{array}{c}\text { Apnea } \\
V^{\prime} \mathrm{O}_{2}\end{array}$ & $\begin{array}{c}\text { Control } \\
\dot{V}_{\mathrm{CO}_{2}}\end{array}$ & $\begin{array}{c}\text { Apnea } \\
V_{\mathrm{CO}_{2}}^{\prime}\end{array}$ & Control & Apnea \\
\hline TM & +5.3 & 110 & 310 & -85 & -194 & 0.77 & 0.70 \\
\hline " & -5.3 & 111 & 365 & -88 & -204 & 0.79 & 0.56 \\
\hline YH & +5.3 & 74 & 225 & -59 & -157 & 0.80 & 0.70 \\
\hline ", & zero & 80 & 283 & -69 & -205 & 0.75 & 0.72 \\
\hline PP & +5.3 & 81 & 210 & -69 & -154 & 0.85 & 0.73 \\
\hline ," & zero & 85 & 193 & -63 & -154 & 0.74 & 0.80 \\
\hline " & -5.3 & 87 & 324 & -71 & -166 & 0.82 & 0.51 \\
\hline
\end{tabular}

The four volumes are in $\mathrm{ml} \mathrm{STPD}$, and both control values and the apnea gas exchange are calculated in 15 -s periods. ${ }^{*}$ The alveolar exchange ratio. ${ }^{* *}$ The intrapulmonic pressure $(5.3 \mathrm{kPa}=40$ Torr $)$.

values, Table 2). The $R$ value fell during apnea-most at negative, intrapulmonic pressure.

\section{Part III}

Stroke volume fell, and HR rose during Valsalva apnea, compared to the control level, whereas smaller changes took place during Mueller apnea (Table 3). The systolic pressure was slightly reduced during both apnea periods, but the size of the MAP include an increased diastolic pressure (Table 3). TPR was increased during Valsalva apnea, and not during Mueller apnea (Table 3). The stroke work of the left ventricle was reduced to half the size during Valsalva apnea, but less during Mueller apnea (W left, Table 3).

\section{DISCUSSION}

The effect of Valsalva and Mueller maneuvers on cardiac output and stroke volume has received only little attention. During Valsalva apnea, the veins running to the right atrium will be compressed, with diminution of the venous return. This leads to a decrease of the sv of the right ventricle and eventually of the left, according to Starlings law of the heart. The predicted decrease in sv during Valsalva apnea was verified with indicator dilution technique (PAulev and WeTterQvist, 1968). The considerable transient fall in MAP and pulse pressure, occurring during the first $5-10 \mathrm{~s}$ of Valsalva apnea, must be accompanied by a fall in impulse frequency from the baroreceptors in the carotid sinuses and from those in the aortic arch. In these baroreceptor regions the increase in external pressure further 
Table 3. Cardiovascular mean values (S.E.M.) in each of three subjects of 8 Mueller apnea and 8 Valsalva apnea periods-all compared with the preceding control phases. Where only single values are available they are shown without S.E.M.

\begin{tabular}{lcc}
\hline & $\begin{array}{c}\text { Mueller } \\
\text { apnea/control }\end{array}$ & $\begin{array}{c}\text { Valsalva } \\
\text { apnea/control }\end{array}$ \\
\hline Subject TM & & \\
Stroke volume $(\mathrm{sv} ; \mathrm{ml})$ & $88(4.5) / 87(1.3)$ & $55(1.8) / 84(1.2)^{*}$ \\
Heart rate $(\mathrm{HR}$; beats/min) & $78(1.8) / 78(0.8)$ & $82(3.8) / 78(1.5)$ \\
Systolic pressure $(\mathrm{kPa})$ & $14.9 / 16.3$ & $14.1 / 16.3$ \\
MAP $(\mathrm{kPa})$ & $12.1 / 12.9$ & $10.0 / 12.9$ \\
TPR $(\mathrm{kPa} \times \mathrm{s} / l=\mathrm{PRU})$ & $106 / 114$ & $159 / 118$ \\
W left $(\mathrm{kJ})$ & $1.065 / 1.122$ & $0.403 / 1.084$ \\
Subject WN & & \\
sv & $91(12) / 89(7)$ & $63(5) / 89(7)^{*}$ \\
HR & $72(1.2) / 70(1.3)$ & $81(2.8) / 76(1.9)$ \\
Systolic pressure & $14.4 / 16.3$ & $14.1 / 16.3$ \\
MAP & $11.2 / 11.9$ & $12.5 / 11.9$ \\
TPR & $104 / 115$ & $116 / 106$ \\
W left & $1.019 / 1.059$ & $0.619 / 1.059$ \\
Subject PP & & \\
sv & $62(4.1) / 75(2.5)$ & $44(2.8) / 72(1.5)^{*}$ \\
HR & $73(1.8) / 65(0.8)$ & $81(1.5) / 65(1.0)$ \\
Systolic pressure & $15.1(1.0) / 15.8(0.3)$ & $14.4(1.4) / 15.9(0.5)$ \\
MAP & $12.0(0.2) / 12.1(0.1)$ & $12.2(0.3) / 12.1(0.2)$ \\
TPR & $172 / 156$ & $191 / 157$ \\
W left & $0.744 / 0.908$ & $0.419 / 0.871$ \\
\hline
\end{tabular}

The average (S.E.M.) intrapulmonic pressure was $-3.2(0.7)$ and $3.1(0.9) \mathrm{kPa}$ during Mueller and Valsalva apnea periods, respectively. ${ }^{*}$ A statistically significant fall in sv.

decreases the transmural arterial pressure (TAP). Thus, the low TAP of the initial Valsalva apnea must have a strong effect on the sensitive sino-aortic baroreceptors, since the medullary vasomotor and cardiac control centers respond with a substantial arteriolar and a venous constriction (PAULEv, 1969). During apnea at TLC and negative, intrapulmonic pressures (Mueller), only minor changes in left ventricular stroke volume and arterial blood pressure were found, but a rise in systemic arterial pressure would add a further stimulus to the baroreceptors, and explain the fall in HR actually observed. At an intrapulmonic pressure of $+1.3 \mathrm{kPa}$, no $\mathrm{HR}$ response is to be expected, since the intrathoracic pressure is of the same value as with the lungs in the normal midposition in the control periods. The predicted heart rates were actually found (PAulev, 1969): The structurally and functionally well-defined sino-aortic baroreceptors of man seem to play an important role during apnea, in particular during apnea with straining, the point being to 


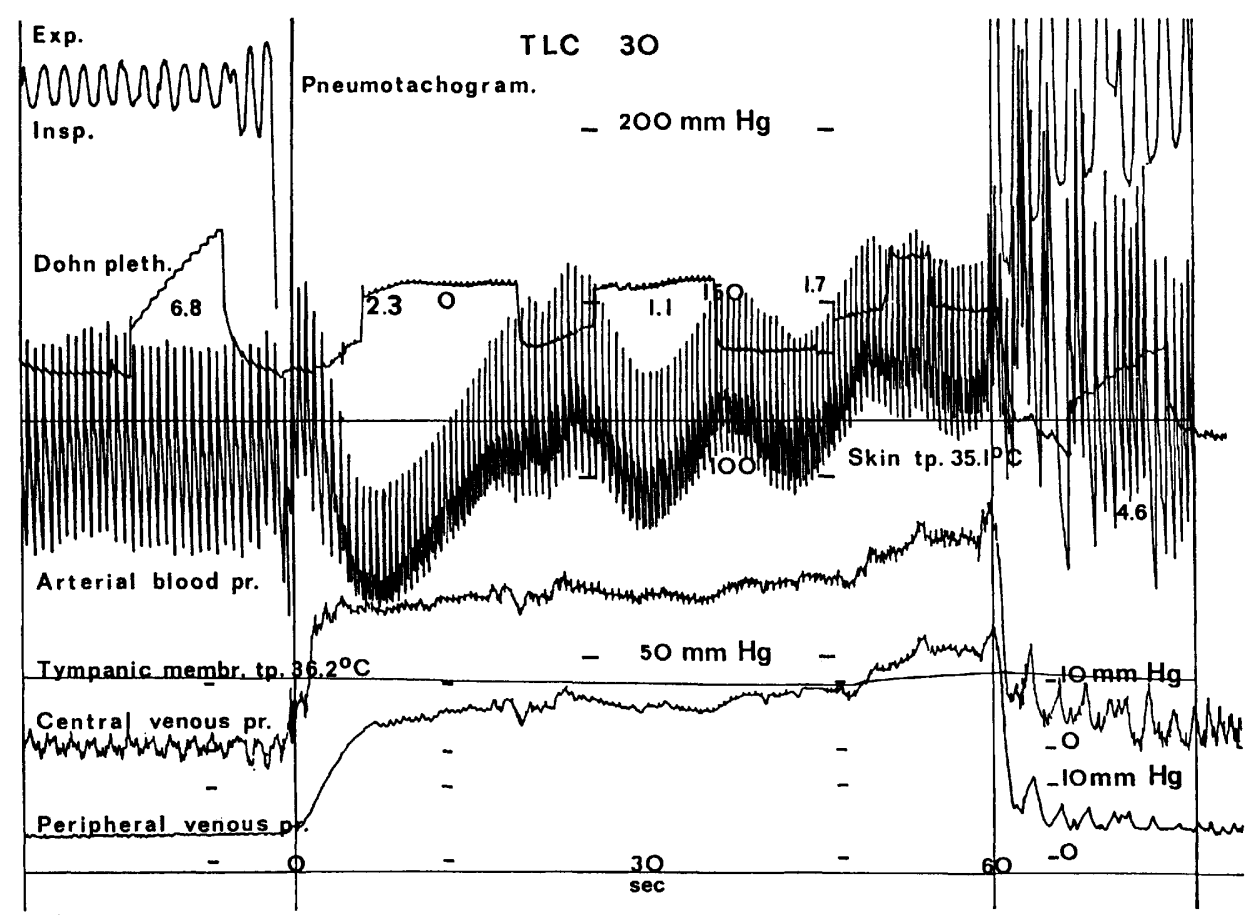

Fig. 3. Simultaneous recording of brachial blood pressure and antebrachial blood pressure and antebrachial blood flow with a segment plethysmograph in subject PP (PAulev, 1969). The apnea was performed following a deep inspiration, and at an intrapulmonic pressure of $4 \mathrm{kPa}(+30$ Torr). On the ordinate, 0 to $10 \mathrm{mmHg}$ are venous pressures; $50,100,150,200 \mathrm{mmHg}$ are arterial pressures. The blood flow values are indicated on the plethysmogram. The temperatures are practically constant.

avoid a substantial fall in blood pressure. The cardiovascular alterations of the initial Valsalva phase were found by others to depend on the magnitude of the pressure of the expiratory strain (CRAIG, 1963; MANTYSAARI et al., 1984). Also the HR effect during face immersion with involuntary Valsalva maneuvers was found to vary with the related elevations of intrathoracic pressure (BJERTNAES et al., 1984). In both of these reports, the changes in HR was explained as arterial baroreceptor reactions. Honde et al. (1987) studied apnea in air and with face immersion, using cardiac impedance technique. The magnitude of the apnea response was almost the same as in air, and when the face was covered by a wet cloth with water $\left(12^{\circ} \mathrm{C}\right)$ dripping from a reservoir. Honda et al. (1987) found no sign of "oxygen conservation" and concluded that the main phenomenon was an "apnea bradycardia." In our study, the stroke volume and the stroke work are clearly reduced during the Valsalva apnea, compared to the Mueller apnea. Yet the Valsalva tachycardia seems to compensate, insufficiently, as judged by both the $\dot{Q}$ - and the sv/HR-ratio. 
The reduced stroke work is a natural consequence of the increase in ventricular afterload during apnea, when the diastolic pressure is increased. During face immersion with apnea, a reduction in myocardial oxygen demand was estimated (BJERTNAES et al., 1984).

The Valsalva maneuver. The immediate rise in brachial artery pressure, following the inspiration before apnea, and the subsequent fall in pulse pressure and MAP, can only be shown by direct recording (Fig. 3), which is taken from a book by one of the authors (PAULEV, 1969). The high transmural pressure during inspiration reduces $H R$ for a few seconds, but then the pressure falls together with a gradually developing rise in HR. During the Valsalva apnea shown here (apnea in air at "TLC"), the fall in arterial pressure elicits a strong, arteriolar constriction with antebrachial blood flow, varying from zero to $1.7 \mathrm{ml}$ of blood per min per $100 \mathrm{~g}$ tissue. The CVP rise from zero up to $3 \mathrm{kPa}$ (30 Torr)-with $2.7 \mathrm{kPa}$ (20 Torr) as an average- during Valsalva, which both verify the intrapulmonic pressure aimed at, and, at the same time, illustrates the difficulties for the subject to keep the pressure steady (Fig. 3). These variations in intrapulmonic pressure during apnea seem to explain the variations recorded in heart rate. Our study has thus confirmed our previous findings of substantial reductions of sv and $\dot{Q}$ during Valsalva maneuvers, and also of the rise in HR and TPR (PAULEV, 1969). The generalized vasoconstriction, and the simultaneous rise in HR (tachycardia) is explainable on the basis of the fall in transmural pressure of the sino-aortic baroreceptors.

The Mueller maneuver. The much smaller changes during Mueller maneuvers result in no change or a large rise in the transmural arterial pressure, compared to the control phase, and it is followed by no change in heart rate or by a fall (called "bradycardia"). The minimum HR reached in our Mueller apnea series was 53 beats/min, which can be compared to the maximum change recorded by BJERTNAES et al. (1984) - from 160 to 52 beats/min during face immersion with apnea, which was considered as a simulated dive. Accordingly, "diving bradycardia" in man has the same magnitude as the "apnea bradycardia" without water studied both here and in previous reports (Paulev, 1968, 1969; Paulev and Wetterqvist, 1968).

Contrary to an organ-specific prolonged use of the oxygen stores, we found that oxygen uptake, as well as carbon dioxide elimination, rose during apnea. This is due to the large alveolar surface area and a better gradient for oxygen diffusion during apnea at TLC. Cardiovascular apnea reactions are explainable in terms of classical physiological mechanisms, protecting the driving pressure and the flow of blood through active organs. These substantial reactions are biologically important in our everyday life above water (lifting, straining during defecations and micturition, etc.), and they should not be considered only a diving phenomenon. Nevertheless, man may still have the capacity to provoke reactions similar to those shown by animals during restrained diving. These reactions may operate in emergency situations (including diving), but for obvious reasons they have seldom been studied by physiologists. 
We are grateful to Mayomi Iino and Kirsten McCord for technical assistance, and to the Scandinavian-Japan Sasakawa foundation, Denmark, for grants to the project.

\section{REFERENCES}

Bjertnaes, L., Hauge, A., KJekshus, J., and Soyland, E. (1984) Cardiovascular responses to face immersion and apnea during steady state muscle exercise. Acta Physiol. Scand., 120: $605-612$.

BuIx, A. S. and Folkow, B. (1983) Cardiovascular adjustments to diving in mammals and birds. In: Handbook of Physiology; The Cardiovascular System, Am. Physiol. Soc., Washington, D.C., Sect. 2, Vol. III, Chapt. 25, pp. 917-945.

Craig, A. B., Jr. (1963) Heart rate responses to apneic underwater diving and to breath holding in man. J. Appl. Physiol., 18: 854-862.

ElSNER, R. and SCHOlandeR, P. F. (1965) Circulatory adaptations to diving in animals and men. In: Physiology of Breath-Hold Diving and the Ama of Japan, ed. by RAHN, H. and Yokoyama, T., Publ. 1341: Natl. Acad. Sci., Natl. Res. Council., Washington, D.C., pp. 281-294.

Honda, Y., Sakakibara, Y., Morikawa, T., Tanaka, Y., and Nakamura, W. (1987) Simultaneous observation in respiratory and circulatory responses after facial application of ice bag in man. In: The Proceedings of the 9th Symposium on Underwater and Hyperbaric Physiology.

HoNG, S. K. (1987) Man as a breath-hold diver. In: Proceeding of the Vancouver Island Satellite Symposium of the IUPS Congress of "Diving Physiology and Hypometabolism."

IRVING, L. (1964) Comparative anatomy and physiology of gas transport mechanisms. In: Handbook of Physiology. Respiration., Am. Physiol. Soc., Washington, D.C., Sect. 3, Vol. I, Chapt. 5, pp. 177-212.

Mantysaari, M., Antila, K., and Peltoren, T. (1984) Relationship between the changes in heart rate and cardiac output during the Valsalva maneuver. Acta Physiol. Scand. (Suppl.), 537: 45-49.

Miyamoto, Y., Tamura, T., and Mikami, T. (1981) Automatic determination of cardiac output using an impedance plethysmograph. Biotelem. Patient Monit., 8: 189-203.

Miyamoto, Y., Hiura, T., Tamura, T., Nakamura, T., Higuchi, J., and Mikami, T. (1982) Dynamics of cardiac, respiratory and metabolic function in man in response to step work load. J. Appl. Physiol., 52: 1198-1208.

Muzi, M., Ebert, T. J., Tristani, F. E., Jeuter, B. C., Barney, J. A., and Smith, J. J. (1985) Determination of cardiac output using ensemble-averaged impedance cardiograms. J. Appl. Physiol., 58: 200-205.

Olsen, C. R., Fanestil, D. D., and Scolander, P. F. (1962) Some effects of breath holding and apneic underwater diving on the cardiac rhythm in man. J. Appl. Physiol., 17: 461466.

Paulev, P. (1968) Cardiac rhythm during breath-holding and water immersion in man. Acta Physiol. Scand., 73: 139-150.

Paulev, P. (1969) Respiratory and cardiovascular effects of breath-holding. Dissertation, Univ. of Copenhagen. Also published: Acta Physiol. Scand. (Suppl.), 324, 1-116.

Paulev, P. and WetTerqvist, H. (1968) Cardiac output during breath-holding in man. Scand. J. Clin. Lab. Invest., 22: 115-123. 